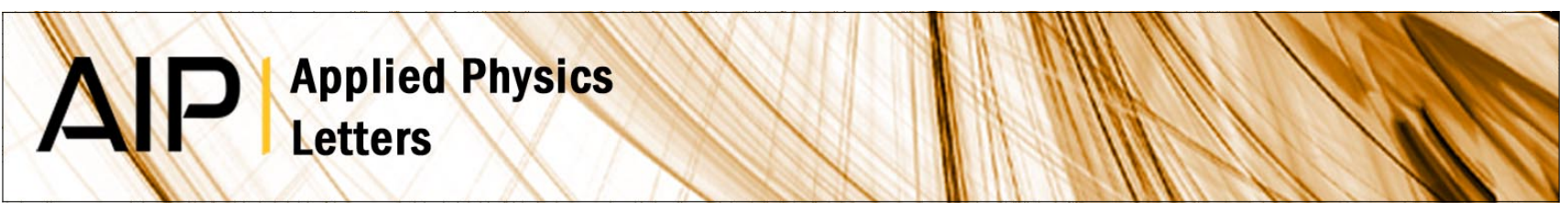

\title{
Gallium phosphide photonic crystal nanocavities in the visible
}

Kelley Rivoire, Andrei Faraon, and Jelena Vuckovic

Citation: Appl. Phys. Lett. 93, 063103 (2008); doi: 10.1063/1.2971200

View online: http://dx.doi.org/10.1063/1.2971200

View Table of Contents: http://apl.aip.org/resource/1/APPLAB/v93/i6

Published by the American Institute of Physics.

\section{Related Articles}

High-Q AIN photonic crystal nanobeam cavities fabricated by layer transfer Appl. Phys. Lett. 101, 101106 (2012)

Experimental demonstration of surface morphology independent electromagnetic chiral edge states originated from magnetic plasmon resonance

Appl. Phys. Lett. 101, 081912 (2012)

Optimized optomechanical crystal cavity with acoustic radiation shield

Appl. Phys. Lett. 101, 081115 (2012)

Development of metamaterials with desired broadband optical properties

Appl. Phys. Lett. 101, 071907 (2012)

Enhancement of spontaneous emission in three-dimensional low refractive-index photonic crystals with designed defects

Appl. Phys. Lett. 101, 071109 (2012)

\section{Additional information on Appl. Phys. Lett.}

Journal Homepage: http://apl.aip.org/

Journal Information: http://apl.aip.org/about/about_the_journal

Top downloads: http://apl.aip.org/features/most_downloaded

Information for Authors: http://apl.aip.org/authors

\section{ADVERTISEMENT}

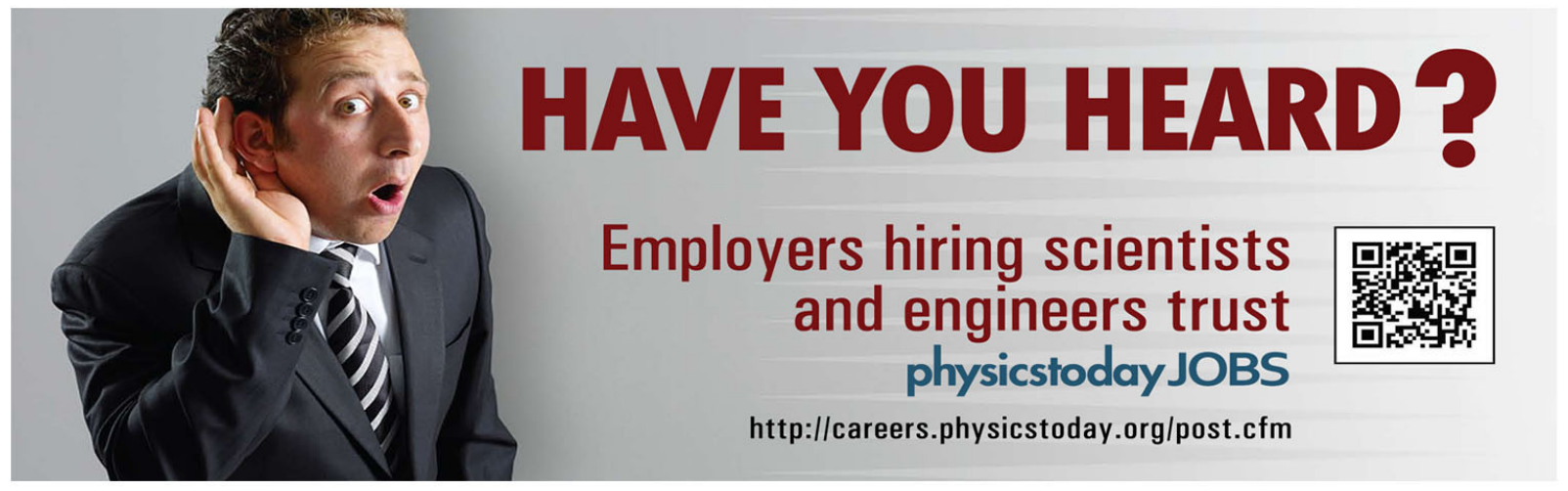




\title{
Gallium phosphide photonic crystal nanocavities in the visible
}

\author{
Kelley Rivoire, ${ }^{\text {a) }}$ Andrei Faraon, and Jelena Vuckovic \\ E. L. Ginzton Laboratory, Stanford University, Stanford, California 94305-4085, USA
}

(Received 2 July 2008; accepted 28 July 2008; published online 14 August 2008)

\begin{abstract}
Photonic crystal nanocavities at visible wavelengths are fabricated in a high refractive index $(n$ >3.2) gallium phosphide membrane. The cavities are probed via a cross-polarized reflectivity measurement and show resonances at wavelengths as low as $645 \mathrm{~nm}$ at room temperature, with quality factors between 500 and 1700 for modes with volumes $0.7(\lambda / n)^{3}$. These structures could be employed for submicron scale optoelectronic devices in the visible, and for coupling to emitters with resonances in the visible such as nitrogen vacancy centers, and biomolecules and organic molecules. (C) 2008 American Institute of Physics. [DOI: 10.1063/1.2971200]
\end{abstract}

Photonic crystal nanocavities confine light in ultrasmall volumes, making them ideal for low-power, on-chip optoelectronic devices, ${ }^{1-3}$ as well for exploring fundamental light-matter interactions. ${ }^{4,5}$ Most experimental work in photonic crystal cavities has been done at telecom bands. ${ }^{5}$ This has been facilitated by well established fabrication procedures for semiconductors such as silicon and gallium arsenide, which have band gaps in the infrared. Photonic crystal devices operating in the visible part of the spectrum, however, could serve as light sources and spectroscopic devices operating below $750 \mathrm{~nm}$. Photonic crystal cavities with resonances in the visible could also be coupled to light emitters such as nitrogen vacancy centers, ${ }^{6,7}$ fluorescent molecules, ${ }^{8}$ and visible colloidal quantum dots. ${ }^{9}$

To date, most photonic crystal devices operating in the visible have been fabricated in one of the following three material systems: gallium nitride, silicon nitride, and AlGaInP. While devices with wavelengths as short as blue can be fabricated in gallium nitride, ${ }^{10,11}$ fabrication processes in this system are difficult. The low refractive index of gallium nitride $(n=2.4)$ limits the device quality factors. ${ }^{12}$ Low refractive index is also the main drawback of the silicon nitride system. ${ }^{13,14}$ Chen et al. ${ }^{15}$ and Zhang et al. ${ }^{16}$ have fabricated AlGaInP-based photonic crystals in the red with InGaP quantum wells. This system, however, requires precise control over the fraction of each material in the quaternary system to obtain the proper band gap and lattice constant, and is lattice matched to a GaAs substrate, which is absorbing in the visible.

In this letter, we show that photonic crystal devices at visible wavelengths can be fabricated in gallium phosphide. Gallium phosphide is a high refractive index $(n=3.25$ at 700 $\mathrm{nm}, n=3.44$ at $555 \mathrm{~nm}$ at room temperature ${ }^{17}$ ) III-V semiconductor with an indirect band gap at $555 \mathrm{~nm}$ at room temperature. Although gallium phosphide is not typically used for devices requiring very high brightness because of its indirect band gap, incorporating InP quantum dots or quantum wells, ${ }^{18}$ or colloidal quantum dots should greatly increase the quantum efficiency. Additionally, gallium phosphide has a relatively low surface recombination rate, estimated to be roughly an order of magnitude less than that of gallium arsenide, ${ }^{19}$ because of its high phosphorus content, which has a passivating effect. ${ }^{20}$ Surface recombination is particularly

${ }^{a)}$ Electronic mail: krivoire@stanford.edu. important for photonic crystal devices because of the additional exposed surface area from etched air holes.

The linear three-hole defect $^{21}$ (L3) cavities were first simulated and then fabricated in a $140 \mathrm{~nm}$ thick GaP membrane (Fig. 1). Simulation results indicate that quality factors above 10000 can be obtained for this type of cavity (three holes removed, holes next to cavity displaced outward by $0.15 a$ ) using a triangular lattice of lattice constant $a$ with slab thickness $d / a=0.55$ and hole radius $r / a=0.3$ with no other design modifications. The electric field profile for the fundamental L3 cavity mode is shown in Fig. 1(b).

To fabricate these structures, the $140 \mathrm{~nm}$ thick GaP membrane was grown on the top of a $1 \mu \mathrm{m}$ thick sacrificial $\mathrm{AlGaP}$ layer. The patterns were first defined in ZEP520 photoresist by electron-beam lithography and then transferred into the $\mathrm{GaP}$ membrane by a chlorine-based reactive ion etch. Excess photoresist was removed with oxygen plasma. The sacrificial layer was undercut with hydrofluoric acid to yield suspended membrane structures with high index contrast. Scanning electron microscope images of one of the fabricated membranes can be seen in Fig. 1(a).

The fabricated photonic crystal resonators were probed using a confocal cross-polarized reflectivity measurement ${ }^{22,23}$ technique, as depicted in Fig. 2(a). This technique uses polarization control to achieve a high signalto-noise ratio and allows probing of cavities with no internal light source. A vertically polarized $(V)$ probe is directed through a polarizing beam splitter (PBS) and a half wave plate (HWP) onto the photonic crystal cavity, which has a mode that is also vertically polarized $(V)$. The reflected output is observed through the PBS, which acts as a horizontal $(H)$ polarizer. The primary polarization of the cavity mode is (a)

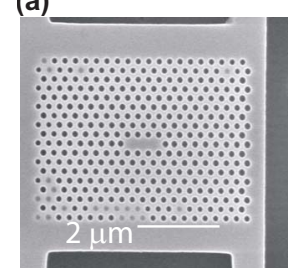

(b)

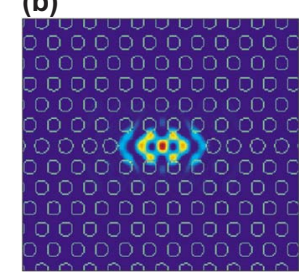

FIG. 1. (Color online) (a) Scanning electron microscope image of a fabricated photonic crystal membrane after undercut of sacrificial layer with lattice constant $a=246 \mathrm{~nm}$, hole radius $r / a=0.32$, and slab thickness $d / a$ $=0.57$. (b) Finite difference time domain simulation of electric field intensity inside the cavity for the high $Q$ mode. 


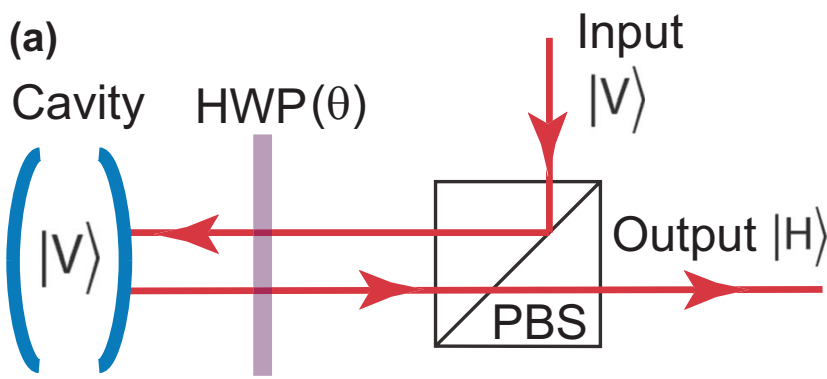

(b)

(c)
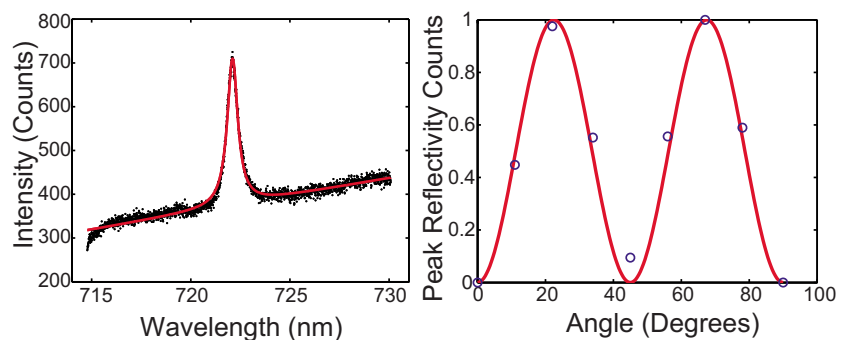

FIG. 2. (Color online) (a) Experimental setup. The $V$-polarized cavity is probed in a cross-polarized setup using a PBS and a HWP. The cavity signal observed at the output follows a $\sin (4 \theta)$ dependence where $\theta$ is the HWP setting ( $\theta=0$ corresponds to $V$ polarization). (b) Spectrum of a cavity measured with configuration of (a) with HWP at $\theta=67^{\circ}$. The cavity resonance is at $722 \mathrm{~nm}$ at room temperature. A fit to a Lorentzian (solid line) gives $Q$ $=1100$. (d) (Circles) Normalized counts in cavity peak as a function of HWP angle (background subtracted). (Line) Fit with $\sin (4 \theta)$.

vertical $(V)$. When the angle between the HWP fast axis and the vertical direction $(\theta)$ is set to zero, the cavity-coupled light is reflected with $V$ polarization and does not transmit through the beam splitter. Rotation of the HWP allows part of the cavity-coupled light to be transmitted at the PBS into the output port with intensity following a $\sin (4 \theta)$ dependence [Figs. 2(b) and 2(c)]. A tungsten halogen lamp source was used as a wide broadband signal at the input. The output field was measured using a spectrometer with a liquid nitrogen cooled charge coupled device.

To fabricate cavities with resonant wavelengths spanning a large interval, lattice constant a $(210 \mathrm{~nm}<a<246 \mathrm{~nm})$ and hole radius $(0.25<r / a<0.35)$ were modified. These changes in the fabrication parameters resulted in cavities with resonance wavelength spanning from 645 to $750 \mathrm{~nm}$, as shown in Fig. 3. (Plots in Fig. 3 are shown with background subtracted.) The measured resonances have shorter wavelengths than predicted from finite difference time domain simulations (by about $0.05 a / \lambda$ ). We believe this difference is a result of imperfect selectivity between the sacrificial layer and membrane during the wet etch step, which reduces the membrane thickness.

Quality factors measured experimentally are between 500 and 1700 - about one order of magnitude smaller than predicted by simulations because of imperfections introduced in the fabrication process. However, these $Q$ 's can still enable a significant Purcell effect, up to 185 , as mode volume is only $0.7(\lambda / n)^{3}$. While these quality factors are similar to those measured at visible wavelengths in optimized structures with lower index contrast, ${ }^{13}$ improving fabrication and using modified designs with simulated $Q$ values more than a order of magnitude higher ${ }^{24}$ should yield structures with much higher $Q$. Additionally, a wider photonic band gap al-

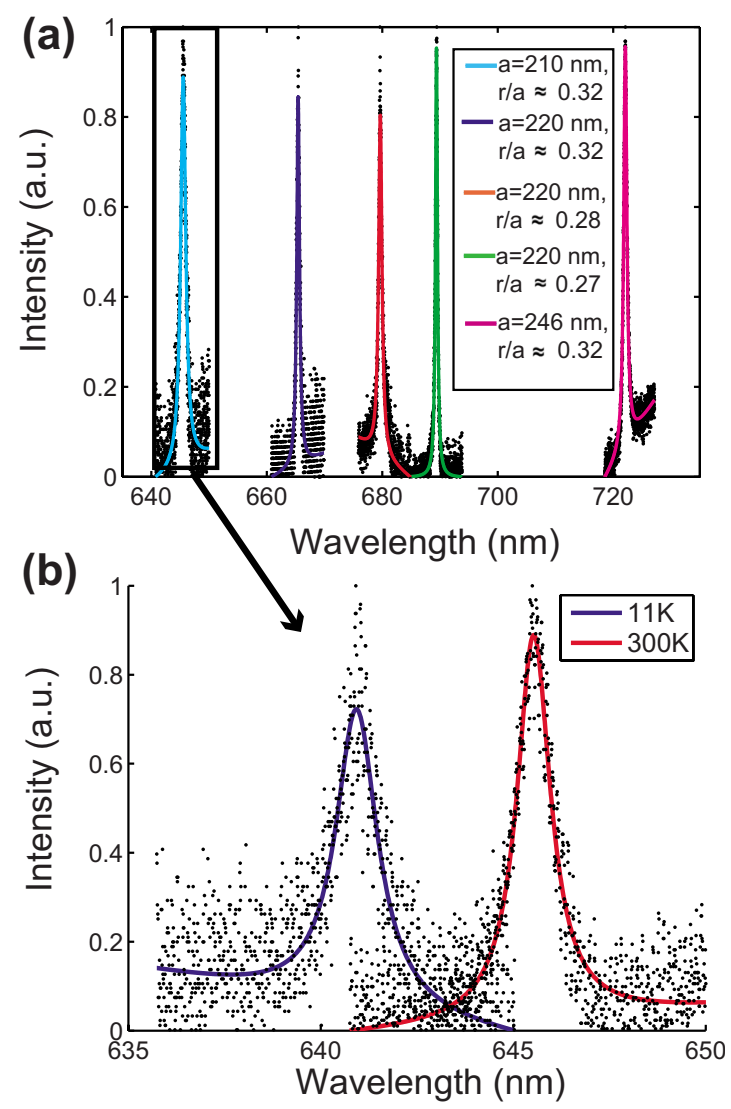

FIG. 3. (Color online) (a) Shift in photonic crystal resonance at room temperature as $a$ and $r / a$ are changed. Solid lines are fits to Lorentzians. (b) Cavity resonance measured at room temperature (right) and low temperature (left). Solid lines indicate fits to Lorentzians.

lows more flexibility in cavity design, is more robust to fabrication variations, and is more suitable for any experiments in which the undercut structures are back filled with a low index material. ${ }^{25}$

The shortest resonant cavity wavelength observed at room temperature was $645 \mathrm{~nm}$ with a quality factor of 610 . The resonance wavelength depends on the temperaturedependent refractive index of the membrane. To shift the cavity resonance to lower wavelengths, the sample was cooled to $11 \mathrm{~K}$ in a continuous flow liquid helium cryostat. The change in temperature caused the resonance to shift from 645 to $641 \mathrm{~nm}$ (in the red wavelength range) and the quality factor to decrease to 490, as shown in Fig. 3. With improved fabrication, devices operating close to the $555 \mathrm{~nm}$ band gap of gallium phosphide could be made; such devices could be used as green light-emitting diodes (LEDs) and lasers.

In summary, we demonstrate a gallium phosphide-based materials system for photonic crystals in the visible. We probe L3 cavities in reflectivity and observe resonances from 645 to $750 \mathrm{~nm}$ at room temperature with quality factors as high as 1700 .

We expect this materials system to allow access to a range of photonic crystal devices in the visible, including LEDs and lasers. Additionally, the gallium phosphide system will provide opportunities to couple photonic crystal cavities to emitters in the visible such as colloidal quantum dots, nitrogen vacancy centers, biomolecules, or organic molecules. 
Financial support was provided by the MARCO Interconnect Focus Center, CIS Seed Funding, and the NSF graduate fellowship. This work was performed in part at the Stanford Nanofabrication Facility of NNIN supported by the National Science Foundation under Grant No. ECS-9731293.

${ }^{1}$ H. Altug, D. Englund, and J. Vuckovic, Nat. Phys. 2, 484 (2006).

${ }^{2}$ I. Fushman, E. Waks, D. Englund, N. Stoltz, P. Petroff, and J. Vuckovic, Appl. Phys. Lett. 90, 091118 (2007).

${ }^{3}$ T. Tanabe, M. Notomi, S. Mitsugi, A. Shinya, and E. Kuramochi, Appl. Phys. Lett. 87, 151112 (2005).

${ }^{4}$ J. Vuckovic and Y. Yamamoto, Appl. Phys. Lett. 82, 2374 (2003).

${ }^{5}$ S. Noda, M. Fujita, and T. Asano, Nat. Photonics 1, 449 (2007).

${ }^{6}$ L. Childress, M. G. Dutt, J. Taylor, A. Zibrov, F. Jelezko, J. Wrachtrup, P. Hemmer, and M. Lukin, Science 314, 281 (2006).

${ }^{7}$ A. Gruber, A. Drabenstedt, C. Tietz, L. Fleury, J. Wrachtrup, and C. von Borczyskowski, Science 276, 2012 (1997).

${ }^{8}$ B. Lounis and W. E. Moerner, Nature (London) 407, 491 (2000).

${ }^{9}$ B. Lounis, H. A. Bechtela, D. Gerionc, P. Alivisatos, and W. E. Moerner, Chem. Phys. Lett. 329, 399 (2000).

${ }^{10}$ Y. Choi, K. Hennessy, R. Sharma, E. Haberer, Y. Gao, S. DenBaars, S. Nakamura, and E. Hu, Appl. Phys. Lett. 87, 243101 (2005).

${ }^{11}$ C. Lai, P. Yu, T. Wang, H. Kuo, T. Lu, S. Wang, and C. Lee, Appl. Phys. Lett. 91, 041101 (2007).
${ }^{12}$ A. Adawi, A. Chalcraft, D. Whittaker, and D. Lidzey, Opt. Express 15, 14299 (2007).

${ }^{13}$ M. Barth, J. Kouba, J. Stingl, B. Lochel, and O. Benson, Opt. Express 15, 17231 (2007).

${ }^{14}$ M. Makarova, J. Vuckovic, H. Sanda, and Y. Nishi, Appl. Phys. Lett. 89, 221101 (2006)

${ }^{15}$ A. Chen, S. Chua, G. Xing, W. Ji, X. Zhang, J. Dong, L. Jian, and E. Fitzgerald, Appl. Phys. Lett. 90, 011113 (2007).

${ }^{16}$ Z. Zhang, T. Yoshie, X. Zhu, J. Xu, and A. Scherer, Appl. Phys. Lett. 89, 071102 (2006).

${ }^{17}$ D. Nelson and E. Turner, J. Appl. Phys. 39, 3337 (1968).

${ }^{18}$ F. Hatami, W. Masselink, and J. Harris, Nanotechnology 17, 3703 (2006)

${ }^{19}$ A. Baca and C. Ashby, Fabrication of GaAs Devices (Institution of Electrical Engineers, 2005), p. 86.

${ }^{20}$ G. Wang, T. Ogawa, K. Murase, K. Hori, T. Soga, B. Zhang, G. Zhao, H. Ishikawa, T. Egawa, T. Jimbo, and M. Umeno, Jpn. J. Appl. Phys., Part 1 40, 4781 (2001).

${ }^{21}$ Y. Akahane, T. Asano, B. Song, and S. Noda, Nature (London) 425, 944 (2003).

${ }^{22} \mathrm{H}$. Altug and J. Vuckovic, Opt. Lett. 30, 982 (2005).

${ }^{23}$ D. Englund, A. Faraon, I. Fushman, N. Stoltz, P. Petroff, and J. Vuckovic, Nature (London) 450, 857 (2007).

${ }^{24}$ D. Englund, I. Fushman, and J. Vuckovic, Opt. Express 13, 5961 (2005)

${ }^{25}$ M. Loncar, A. Scherer, and Y. Qiu, Appl. Phys. Lett. 82, 4648 (2003). 